\title{
REVIEW \\ Selection and work-up for liver transplantation
}

M Zuckermann, J A Loveland

The evaluation of the liver transplantation candidate is intended to confirm the indication for transplantation, determine the severity of disease, exclude contra-indications, optimise pre-transplantation care and candidate condition, and educate the patient and family on post-procedure expectations. This article is intended as a guide for the appropriate selection and work-up of patients for liver transplantation.

S Afr Med J 2012;102(11):876-878. DOI:10.7196/SAMJ.6146

\section{Candidate evaluation}

The primary goal of evaluating patients for liver transplantation (LT) is to identify appropriate candidates and establish a pre-transplantation plan. Prospective patients must first be referred to a transplantation centre for evaluation and, if deemed suitable, for work-up, with a view to being listed for transplantation. South Africa has centres offering LT in Johannesburg and Cape Town. Typically, referred patients are evaluated by a clinician, and further evaluations and tests are performed before the patient is discussed at a multidisciplinary transplantation meeting. Initiating the referral early in the course of disease facilitates improved outcomes, by allowing earlier LT before the establishment of end-stage disease. It is also well described that patients awaiting transplantation (and their families) demonstrate better psychological adaptation when the entire spectrum of issues is approached by a multidisciplinary clinical team. ${ }^{1}$ It is not uncommon for children to exhibit behavioural problems, depression, poor social adaptation and non-compliance after transplantation, which can be moderated by early counselling after referral. ${ }^{2}$

\section{Purpose and policy}

Organ availability is the rate-limiting step regarding successful transplantation and a reduction in waiting-list mortality. This is particularly applicable in centres that do not have related living donor programmes, but rely solely on deceased donor organs. Consequently, the decision-making and selection process must be transparent, and a consistent set of evidence-based criteria must be applied to determine whether selection for transplantation is appropriate.

\section{Indications}

Borne out by our experience, approximately $50 \%$ of paediatric patients requiring LT have biliary atresia. ${ }^{2}$ However, the indications for LT fall into 5 major categories of liver disease: (i) cholestatic diseases; (ii) metabolic disorders; (iii) fulminant liver failure; (iv) auto-immune hepatitis; and $(v)$ liver tumours.

Michele Zuckermann and Jerome Loveland, hepatologist and surgeon respectively, both work in the Department of Paediatric Surgery, Chris Hani Baragwanath Academic Hospital, and Transplant Division, Wits Donald Gordon Medical Centre, both affiliated to the University of the Witwatersrand, Johannesburg, South Africa.

\section{General listing criteria}

Infants and children should be listed for LT when there is evidence that hepatic decompensation has occurred, is imminent, or is inevitable based on the natural history of the disease. Clinical endpoints that determine suitability for transplantation may include one or more of the following: severe cholestasis; portal hypertension with/without variceal bleeding; multiple episodes of ascending cholangitis; failure of hepatic synthetic function; malnutrition and failure to thrive; intractable ascites; encephalopathy; unacceptably poor quality of life due to liver disease; and life-threatening complications of stable liver disease, such as hepatopulmonary syndrome.

\section{Pre-LT assessment and work-up}

The first step in evaluating a potential candidate for LT is to determine the severity and prognosis of the liver disease. A subjective clinical assessment is undertaken together with an objective assessment including comprehensive laboratory and radiological evaluations. The aim of this is to: (i) identify contra-indications that would either exclude LT, require discussion on a case-by-case basis or could be optimised; (ii) identify comorbidities or psychosocial factors that reduce the expectation of successful LT; and (iii) determine the wishes of the patient and family regarding LT., ${ }^{1,3}$ This in-depth assessment determines the suitability of the patient for potential listing, allowing better allocation of resources and, ideally, optimising the survival rate of LT recipients.

Current contra-indications in children include: non-resectable extrahepatic malignancy; concomitant end-stage organ failure that cannot be corrected by combined transplantation; uncontrolled sepsis; and irreversible serious neurological damage.

Once transplantation is considered, a specially trained multidisciplinary clinical team meets with the patient and family to assess suitability for LT and to provide further counselling. The team usually includes: a hepatologist; a transplant surgeon; a cardiologist; a pulmonologist; an anaesthesiologist; transplantation co-ordinators and nursing staff; a psychiatrist and/or psychologist; a physiotherapist; a dietician; and a social worker.

The general work-up is as follows, although specific additional testing may be performed on a case-by-case basis:

\section{- Biochemistry}

- Full blood count and grouping

- Qualitative liver function tests, including synthetic function (albumin and international normalised ratio (INR))

- Urea and electrolytes, with urine microscopy, culture and sensitivities

- Alpha-fetoprotein

- Vitamins A, E and D 
- Serology: hepatitis A, B, C, varicella, HIV, cytomegalovirus, Epstein-Barr virus, herpes simplex virus (HSV) and measles.

- Radiology

- Chest X-ray - abdominal ultrasound (Duplex Doppler), looking specifically at: patency of portal vein with direction of flow; presence of ascites; vascular anatomy of coeliac axis, including hepatic artery resistance index; splenic size; and additional indicators of portal hypertension

- Magnetic resonance angiography, or angiography if vascular anatomy is uncertain (to be discussed with the surgeons).

- Cardiology

- Electrocardiogram and echocardiogram.

- Respiratory

- Cough swab or tracheal aspirate for acid-fast bacilli and culture

- Oxygen saturations

- Respiratory function tests in selected patients.

- Anthropometry

- Immunisation record

- Dental review

- Assessment of the severity of liver disease

- Upper gastro-intestinal endoscopy (if there is evidence of variceal bleeding)

- Liver biopsy to assess hepatic architecture

- Calculation of paediatric hepatology score (Paediatric EndStage Liver Disease (PELD) score - see below).

\section{Listing of candidates}

On completion of the work-up, the patient and results are considered by the candidate's selection committee for a decision regarding suitability for LT. This committee consists of the listed multidisciplinary team and may include the patient's primary care physician. While the structure of this committee may vary substantially between centres, ${ }^{4}$ the process is uniform and primarily involves inductive reasoning to review suitability for LT and possible reasons for exclusion. The latter include systemic comorbidities, patients being 'not sick enough' or 'too sick', and other psychosocial barriers. There is scant information on the quality of life of paediatric LT recipients and their families. Results suggest that psychological support should be made available to both, before and after the operation. Particular attention should be paid to the partners of related living donors and the siblings of affected children to minimise secondary phenomena including marital difficulties, sibling neglect, and other psychological problems before and after LT.

The following questions are posed to the committee before listing a patient for LT:

- Does the patient need LT as therapy for disease?

- Have the indications and contra-indications been assessed properly?

- What is the surgical risk?

- Is the patient's medical condition such to allow tolerance of the procedure and post-operative course?

- What are the chances of recurrent disease affecting graft and patient survival?

\section{Prioritisation}

The allocation criteria for a resource as scarce as a donor liver became crucial in 1999 when it was shown that waiting time - a previous listing criterion - constituted a poor predictor of pre-transplant mortality. An allocation score centred on objective parameters and based on continuous scale measuring of the severity of end-stage liver disease was required. ${ }^{5}$
The liver allocation system, implemented by the Organ Procurement Transplantation Network in the USA in 2002, is based primarily on the severity of liver disease, assessed by the Model for End-stage Liver Disease (MELD) for adults and PELD for paediatric patients with chronic liver disease. ${ }^{6,7}$ The system employs risk determination based on a 3-month pretransplantation assessment, and quantifies the risk of death within the 3 months post transplantation (the higher the score, the higher the mortality). The PELD model, based on analyses of data from the Studies of Pediatric Liver Transplantation (SPLIT), has been shown retrospectively to be predictive of waiting list mortality in paediatric patients. The PELD score, derived from bilirubin, albumin, INR, growth failure and patient age when first placed on the waiting list, may be calculated with a tool available on the United Network for Organ Sharing (UNOS) website. ${ }^{8}$

This system is a step towards a more precise and accountable means of ranking patients and may contribute to reduced waiting times and pre-transplantation mortality among children with advanced liver disease - rather than allocating organs to patients who have waited longer but are more stable. The PELD score has not been proven to be a successful predictor of post-transplantation outcome, but has also not been shown to adversely affect results. ${ }^{7,9,10}$

\section{Waiting period and pre-transplantation care}

Optimising the clinical status of the child on the waiting list is essential, particularly as waiting times are becoming longer and, unfortunately, there are many more potential recipients than donors. Aggressive medical management is often required to treat the major complications of liver failure: intractable ascites; variceal bleeding; and hepatorenal syndrome. The management of malnutrition is the most important contribution of paediatric hepatologists and dieticians to patient management. Nutrition is one of few variables, known to affect both pre- and post-transplantation outcome, that can be prevented or ameliorated. Better-nourished children have decreased mortality, a lower infection risk and fewer post-operative surgical complications. ${ }^{11-13}$

Particular attention is also paid to immunisation. If feasible, live vaccines are administered before transplantation (varicella with measles, mumps and rubella, if aged $>6$ months) and caregivers are given advice on completing other vaccines, such as pneumococcal and hepatitis A and B. Candidates are suspended from the transplantation waiting list for 2 weeks following vaccination with live virus vaccines.

\section{Outcome and graft survival following LT}

The overall results of paediatric LT are encouraging. UNOS reported on 9064 children transplanted between 1997 and 2004, with 1-year patient and graft survival rates of $86 \%$ and $78 \%$ among children aged 1 - 5 years. ${ }^{14,15}$ The SPLIT registry report on 1611 patients showed 1 -year patient and graft survival rates of $88 \%$ and $82 \%$, respectively. ${ }^{16}$ Age at diagnosis, severity of illness, and possibly the technical variants of grafts utilised (reduced-size and split grafts), may be associated with increased morbidity and decreased overall survival. ${ }^{17}$ $\mathrm{Ng}$ et al. reported second and third transplantation rates of $12 \%$ and $2 \%$, respectively, $5 \%$ chronic rejection, and $6 \%$ post-transplant lymphoproliferative disease in their paediatric LT cohort. ${ }^{18}$ Although tests of graft function were preserved in $90 \%$ of 5 -year survivors in the cohort, one-third of children did not have complete normalisation of liver enzymes, suggesting ongoing graft inflammation. ${ }^{18}$

Most long-term survivors of paediatric LT retain good graft function, but may have chronic medical conditions and post- 


\section{FORUM}

transplantation complications. LT success is determined by more than graft survival rates; ongoing management requires the involvement and commitment of healthcare providers within and beyond transplantation teams.

\section{Living donor liver transplantation}

Introduced in 1989, living donor liver transplantation (LDLT) has developed as an alternative to deceased donor transplantation, to overcome the critical organ shortage, particularly in Asia. Living donor transplantation in children is common practice worldwide, and achieves results comparable with those performed with deceased donor organs. ${ }^{19}$ Biliary atresia is an indication for LDLT, and caregivers frequently question the possibility of using this approach early in the course of LT work-up.

LDLT raises several ethical and technical considerations for the donor and recipient; the balance between recipient benefit and the risk of donor morbidity and mortality is central to its justification. Donor safety is of utmost concern. The primary donor selection criterion remains voluntary and informed consent, followed by extensive counselling, work-up and evaluation of donor suitability Internationally reported donor morbidity rates range from $0 \%$ to $67 \%$, depending on the individual definition and recognition of morbidity, which undoubtedly correlates with the experience and volumes of individual centres. ${ }^{20}$ The donor mortality rate has been estimated to be between $0.1 \%$ and $0.3 \%$.

Graft and recipient size matching are important to achieve successful outcomes. The metabolic demands of the recipient must be met, providing a sufficient graft size to meet the recipient's needs without compromising the donor's safety. The refinement of surgical technique, together with a greater understanding of the anatomical and physiological differences in LDLT when compared with deceased donor LT, has improved outcome.

The proven or potential benefits of LDLT include: a reduction in waiting time (thereby decreasing waiting list mortality); the selection of appropriate timing for transplantation; the superior quality of the donor liver; and significant expansion of the donor pool. Before the addition of a living donor programme to a transplantation unit, the risk-to-benefit ratio, availability of deceased donor organs, infrastructure, and recipient demands must be considered.

1. Steinman I, Becker BN, Frost AE, et al. Guidelines for the referral and management of patients eligible for solid organ transplant. Transplantation 2001:91189-91203. [http://dx.doi.org/10.1097/00007890200105150-00001]

2. Loveland J, Govender T, Botha J, Britz R. Paediatric liver transplantation in Johannesburg: A report on our initial twenty nine cases and prospects for the future. S Afr Med J 2012;102(4):233-236.

3. Murray KF, Carithers RL Jr; AASLD. AASLD practice guidelines: Evaluation of the patient for liver transplantation. Hepatology 2005;41:1407-1432. [http://dx.doi.org/10.1002/hep.20704]

transplantation. Hepatology 2005;41:1407-1432. [http://dx.doi.org/10.1002/hep.20704]
Volk ML, Biggins SW, Huang MA, et al. Decision making in liver transplant selection committees: A multicenter study. Ann Intern Med 2011;155(8):503-508.
mons

multicenter study. Ann Intern Med 2011;155(8):503-508.
Freeman RB, Wiesner RH, Harper A, et al. The new liver allocation system: Moving toward evidence5. Freeman RB, Wiesner RH, Harper A, et al. The new liver allocation system: Moving toward eviden
based transplantation policy. Liver Transpl 2002;8:851. [http://dx.doi.org/10.1053/ilts.2002.35927] 6. Kamath PS, Wiesner RH, Malinchoc M, et al. A model to predict survival in patients with end stage Kamath PS, Wiesner RH, Malinchoc M, et al. A model to predict survival in patien
liver disease. Hepatology 2001;33:464. [http://dx.doi.org/10.1053/jhep.2001.22172]

7. McDiarmid SV, Anand R, Lindblad AS. The SPLIT Research Group. Development of a pediatric McDiarmid SV, Anand R, Lindblad AS. The SPLIT Research Group. Development of a pediatric
end-stage liver disease score to predict poor outcome in children awaiting liver transplantation. end-stage liver disease score to predict poor outcome in children awaiting liver tra

8. United Network for Organ Sharing. Liver Transplantation. MELD/PELD Calculator. http://www.unos. org/resources/meldPeldCalculator.asp (accessed 16 June 2012).

McDiarmid SV, Merion RM, Dykstra DM, Harper AM. Selection of pediatric candidates under the PELD system. Liver Transpl 2004;10:S23-S30. [http://dx.doi.org/10.1002/lt.20272]

10. Bourdeaux C, Tri TT, Gras J, et al. PELD score and posttransplant outcome in pediatric liver transplant: A retrospective study of 100 recipients. Transplantation 2005;79:1273-1276. [http://dx.doi. org/10.1097/00007890-200505150-00060

11. McDiarmid SV. Management of the pediatric liver transplant patient. Liver Transpl 2001;7(11 Suppl 1):S77-S86. [http://dx.doi.org/10.1053/jlts.2001.28643]

12. Shepherd RW, Chin SE, Cleghorn GJ, et al. Malnutrition in children with chronic liver disease accepted for liver transplantation: Clinical profile and effect on outcome. J Paediatr Child Health 1991;27:295 299. [http://dx.doi.org/10.1111/j.1440-1754.1991.tb02541.x]

13. Moukarzel AA, Najm I, Vargas JV, et al. Effect of nutritional status on outcome of orthotopic liver transplantation in pediatric patients. Transplant Proc 1990;22:1560-1563.

14. The Organ Procurement and Transplantation Network (OPTN). National Data Reports. Richmond, Virginia, USA: OPTN, 2012. http://optn.transplant.hrsa.gov/ (accessed 20 June 2012).

15. Spada M, Riva S, Maggiore G, et al. Pediatric liver transplantation. World J Gastroenterol 2009;15(6):648-674. [http://dx.doi.org/10.3748/wjg.15.648]

16. Diamond IR, Fecteau A, Millis JM, et al. Impact of graft type on outcome in pediatric liver transplantation: A report from Studies of Pediatric Liver Transplantation (SPLIT). Ann Surg 2007;246(2):301-310. [http://dx.doi.org/10.1097/SLA.0b013e3180caa415]

17. Ng V, Anand R, Martz K, Fecteau A. Liver retransplantation in children: A SPLIT database analysi of outcome and predictive factors for survival. Am J Transplant 2008;8(2):386-395. [http://dx.doi.org/ $10.1111 / j .1600-6143.2007 .02056 . x]$

18. Ng VL, Fecteau A, Shepherd R, et al. Outcomes of 5-Year Survivors of Pediatric Liver Transplantation: Report on 461 Children From a North American Multicenter Registry. Pediatrics 2008;122:el1281135. [http://dx.doi.org/10.1542/peds.2008-1363]

19. Jeon H, Lee SG. Living donor liver transplantation. Current Opinion in Organ Transplantation 2010;15:283-287. [http://dx.doi.org/10.1097/MOT.0b013e32833983ee]

20. Broering DC, Wilms C, Bok P, et al. Evolution of Donor Morbidity in Living Related Live Transplantation. A Single-Center Analysis of 165 Cases. Ann Surg 2004:240(6):1013-1026. [http:// dx.doi.org/10.1097/01.sla.0000146146.97485.6c

Accepted 9 September 2012. 